\title{
Medical equipments high precise detection technology basing on morphology-harris operator
}

\author{
Yang-Yang Mei, Hai-Ming Xie, Lu Han, Shi-Jun Guo
}

School of Medical Instrument and Food Engineering, USST, Shanghai, China.

Email: $\underline{\text { myy19860103@,126.com }}$

Received 5 January 2010; revised 8 February 2010; accepted 4 March 2010.

\begin{abstract}
Medical equipments related to life safety of human, it is important to detect by a high precise method. Image mosaic which based on Harris corner operator is a commonly used method in this area; Harris operator has low calculation burden, it is simple and stable, so it is more effective comparing with other feature point extracted operators. But in this algorithm, corner points can only be detected in a single-scale, there may be losing information of corner points, causing corner point location offset, extracting false corner points because of noise. In order to solve this question, the acquired images should be processed by dilation and erosion operation firstly, then do image mosaic. Results show that image noise can be eliminated effectively after those morphological processes, as well as the false positive noise generated by image glitch. The success rate of image mosaic and detection accuracy can be greatly improved through the Morphology-Harris operator. Measurement of precision instruments which based on this new method will improve the measurement accuracy, and the research in this area will promote the further development of machine vision technology.
\end{abstract}

Keywords: Image Mosaic; Harris Operator; Feature Point Extraction; High Precise Detection; Dilation and Erosion

\section{INTRODUCTION}

With the improvement of living standards, people are concerned about the health problems more and more, especially in recent years, surgical accidents have been happening continuously, which make the physicianpatient relationship nervous. In this situation, the controversy focused on medical equipment whether it is qualified or not is increasing, so it is important to detect medical equipment in a high precise and steady way. Traditional detection methods are inefficiency, the accu- racy is often affected by Man-made factors. Revolutionary changes of precision equipment detection have happened after the rapid development of computer. Through computer vision, we can make non-contact detection for medical instruments which not only improves the detection accuracy but also reduces the impact of Man-made factors. However, a number of common machine vision detection equipments which are restricted by the optical field of view can not capture large view field and wide angle images, and if we pursuit large field of view simply, the image resolution will be reduced, the detection accuracy will be reduced too. In this case, some requirements of detection accuracy of medical devices have not been fulfilled. To resolve this problem, this paper presents a method of using image mosaic which based on Morphology-Harris operator to obtain images of the medical instruments, enhancing image resolution and measuring accuracy.

\section{OVERVIEW OF IMAGE MOSAIC}

Image mosaic is a technology which is used to solve the problem of small view field that cannot make a big picture. It uses computer to match several images automatically to merge a large panoramic image, at the same time because of the mosaic of one image from two images, the image resolution has been greatly improved, so it has a wide using range in modern life. In foreign country, the panoramic image mosaic technology which based on the movement was brought by Richard Szeliskj in 1996, it is the representative and becomes a classic image mosaic algorithm [1], Richard Szeliskj became the founders of the field of image mosaic, he proposed theory has become a classic theoretical system, even today there are still many people study his theory of splicing. On this basis, Shmuel Peleg, Benny Rousso and others brought an automatic adaptation image mosaic model in 2000, it will select adaptive model of splicing in accordance with the different camera movements [2]. Then M. Brown published an article entitled Recongising Panoramas on the General Assembly of ICCV in 2003 [3], and image mosaic using of SIFT algorithm [4] 
was first brought in this article, the algorithm will be automatic completed fully and the results are effective. At the same time, the development of image mosaic is growing rapidly in our country too, algorithms which were proposed by Xiao-Rui Wang, Zu-Xun Zhang et al. greatly promoted the development of image mosaic technology.

Image mosaic technology was early used in photographic mapping to integrate a large number of satellite images. In this application, the accuracy of splicing does not ask for much. But, in detection of precision instruments, especially for precision medical equipments, it is the key point; detection accuracy is affected directly by it. The image mosaic model is shown in Figure 1. Image registration is the key of image mosaic; it is closely related to the success of it. At present, the method of image registration, based on feature point, is used commonly and widely. It has several advantages such as low calculation burden, high sensitive and little affected by noise [5].

\section{HARRIS CORNER OPERATION}

In field of image mosaic, points that change obviously are called feature points. Extraction of feature points is the basis of image registration. There is one sort of feature points called corner points, which are changing intensely of gray value or the intersection point of the outline in image, they reflect the important information of image, so we can get the information that is important and ignore information that is secondary by corner points. Harris corner detector is the most commonly used operator in image mosaic.

\subsection{Principle of Harris Corner Operator}

Harris detector was brought forward by C. Harris and M. J. Stephens in 1988 and based on the feature points of image [6]. While this feature detector is usually called corner detector, it is not just the corners selecting, but rather any image location having large gradients in all directions at a predetermined scale [7]. Signals are processed through auto-correlation function and the result is a matrix $M$ of auto-correlation function. Eigenvalue of matrix $M$ is first-order curvature of the correlation function, and the point could be viewed as a corner if two curvature values are high. Only the first-order difference of the gray image is used in Harris, so the operation is simple and is widely used in practice. The principle is to calculate the gray value changes during the window moving along any direction; it is supposed that the small window of center pixel point $(x, y)$ has moved $u$ along the direction

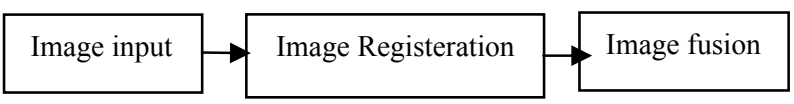

Figure 1. Image mosaic model. of $X$, as well as $V$ along the direction of $Y$, then analytical expression about gray value changes will be given out by Harris, as Eq.1:

$$
E(u, v)=\sum_{x, y} w(x, y)[I(x+u),(y+v)-I(x, y)]^{2}
$$

In the function above, $w(x, y)$ stands for the window function; $[I(x+u),(y+v)-I(x, y)]^{2}$ stands for the gradient of gray image; usually, $w(x, y)$ stands for gauss filter. For a small displacement, Eq.1 can be replaced by Eq.2, as follows:

$$
E(u, v) \cong[u, v] M\left[\begin{array}{l}
u \\
v
\end{array}\right]
$$

$M$ can be expressed as follows:

$$
M=\sum w(x, y)\left[\begin{array}{cc}
I_{x}^{2} & I_{x} I_{y} \\
I_{x} I_{y} & I_{y}^{2}
\end{array}\right]
$$

In Eq.3, $I_{x}$ and $I_{y}$ are the first derivatives of each image channel. $E$ is very similar to the local autocorrelation function and $M$ describes the shape of the origin of auto-correlation function. $\lambda_{1}, \lambda_{2}$ are assumed to be two eigenvalues of $M$, and they will proportion with the local auto-correlation function, constituting a non-variable rotation to $M$ [8]. Through judgment $\lambda_{1}$ and $\lambda_{2}$ the region which changed slowly could be determined, as well as corner and edge.

The maximum value of local region can be defined by Harris feature point:

$$
R=D(M)-k T^{2}(M)
$$

$T(M)$ denotes the matrix trace of $M$, and $T(M)=$ $\lambda_{1}+\lambda_{2} ; D(M)$ stands for the determinant of matrix $M$, and $D(M)=\lambda_{1} \lambda_{2}$. The value of $k$ is usually recommended of $0.02-0.06$.

With the theory of Harris corner detection, several steps could be concluded as follows:

1) Filter every pixel of the image by horizontal and vertical difference operator, through this, $I_{x}$ and $I_{y}$ can be obtained [9].

2) Deal with the four elements of matrix $M$ by gauss filter, a new $M$ will be obtained. Gaussian function is showed below:

$$
\text { Gauss }=\exp \left(-\frac{\left(x^{2}+y^{2}\right)}{2 \partial^{2}}\right)
$$

3) Calculate the interesting value of each pixel by 
function Eq.4.

4) Set the threshold and do non-maximum suppression by it.

The corner points, detected by Harris detection are shown in Figure 2.

\subsection{Disadvantage of Harris Operator and Ameliorate Method}

Harris operator is a widely used feature point extraction operator and has several advantages, but it has some disadvantages too. For example, in this algorithm, corner points can only be detected in a single-scale, there may be losing information of corner points, causing corner point location offset, extracting false corner points because of noise, inaccurate localization to $T$ and diagonal $\mathrm{T}$ and so on.

For tiny medical devices, measurement accuracy is extremely demanding, but because of light, as well as the lens distortion, the image edges will produce some distortion. If the device edge which near the image edge has a glitch, the glitch will have a shape distortion in the two images for mosaic, causing corner point location offset, thus affecting the accuracy of image mosaic. For which we first using morphological dilation and erosion operation to preprocess the image, eliminating the false positive noise generated by image glitch, thus eliminating the inaccurate positioning of the corner points caused by noise, as well as the effect of the corner point location offset, greatly improved the splicing accuracy and detection accuracy.

\section{EXPERIMENT RESULTS}

\subsection{Dilation and Erosion Operation}

According to the illumination of 3.2, first, we process the image through dilation and erosion operation. The result is shown in Figure 3, the left part is the former image, we can see the glitch clearly in the small circle marked on the image, and right half part is image after processing, the glitch is completely disposed.

\subsection{Image Mosaic}

In this part, we use tablet punch to demonstrate the mosaic course. The size of tablet punch determines the weight and density of tablets, the precise of punch is important. First of all, two images of tablet punch using a large field camera can be acquired, then do dilation and erosion operation, the result can be shown in Figure 4. $\mathrm{Be}$ attention that the two images must have some overlapped parts, and this is the basis of image mosaic. Then we can extract the feature points of each image by Harris operator; do image registration by the normalized cross-correlation and image fusion. The result is shown in Figure 5.

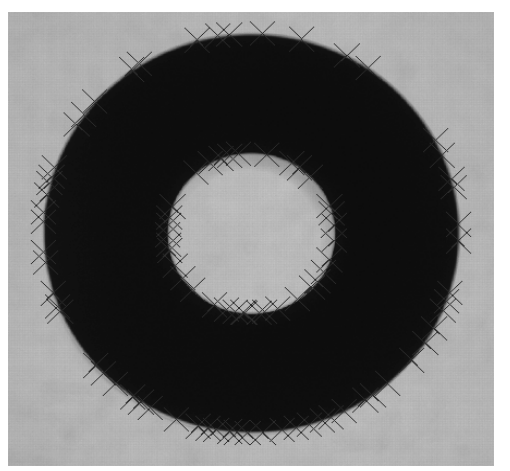

Figure 2. Corner points detected by Harris.

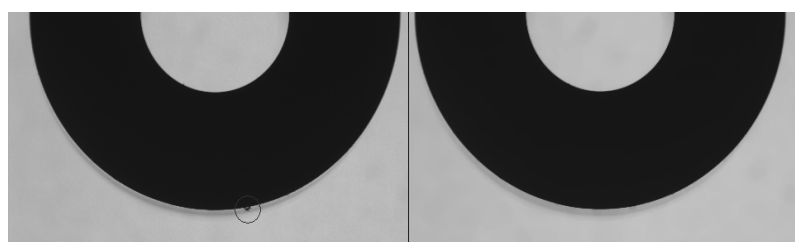

Figure 3. Dilation and erosion processing.

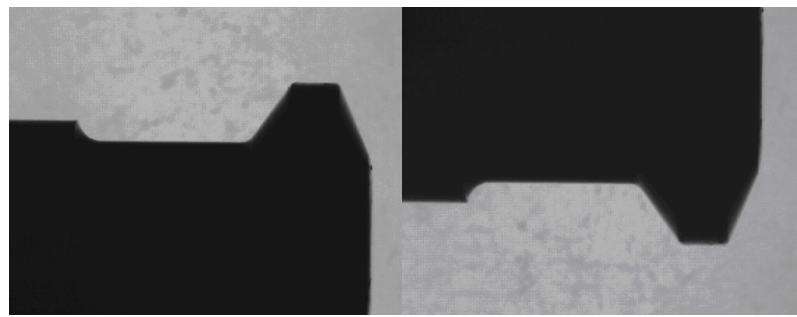

Figure 4. Images waiting for mosaic.

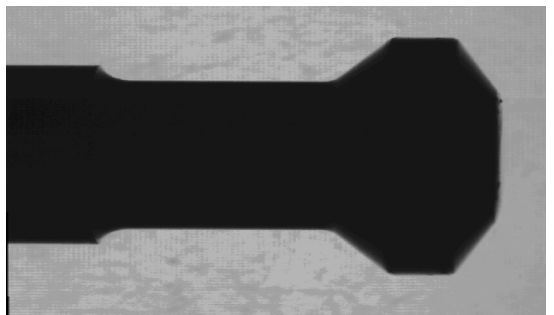

Figure 5. Image after mosaic.

\subsection{Test and Contrast}

After image dilation and erosion processing, mosaic images through registration and fusion, then the width of tablet punch is measured. Here, we test the width in three situations: single image, mosaic image without preprocess, mosaic image by Morphology-Harris Operation. The result images are shown in Figure 6, the left is single image, middle is non-process image result, the right one is mosaic image through Morphology-Harris operator. We found the accuracy of the width in the right 
one is the highest, the left one is the lowest. Size is measured by a method which based on sub-pixel measurement, the pixel value is not an integer number in this way and the measurement result has a higher precise; the value at top left corner of image is every pixel value which is measured after the camera is calibrated using the $30 \mathrm{~mm}$ standard block .

In order to compare the result conveniently, we can see the Table 1 below. In this table, five values can be measured through moving the high of camera in every situation. Absolute difference between single image size and standard size is $0.567 \mathrm{~mm}$; the value between nonpreprocess mosaic image and standard size is $0.430 \mathrm{~mm}$; and the value between Mosaic image of morphological processes and standard size is $0.080 \mathrm{~mm}$. Comparing the results, it is obvious to see that the measurement accuracy of mosaic image after morphological processes is much higher than the single image and Mosaic image of non-preprocess. Through this comparison, the important of dilation and erosion processing of image mosaic in the precision instrument measurement is proved, and the detection accuracy will be improved through image mosaic.

\section{CONCLUSIONS}

Medical equipments related to life safety of human, accurate measurement is particularly important. In this paper, we use the morphology-Harris operator preprocessing images, eliminate the noise caused by lens distortion and light uneven, extract feature point accurately, position accuracy is higher than the untreated image. Through dilation and erosion processes, the accuracy of the mosaic image is improved, simultaneity the cost of additional hardware needed is reduced too. The experiment results has proved that, accuracy of medical device testing which based on morphology-Harris operator of image mosaic fully meets the requirements. This is an efficient, high-precision medical device detection technology, suitable for promotion.

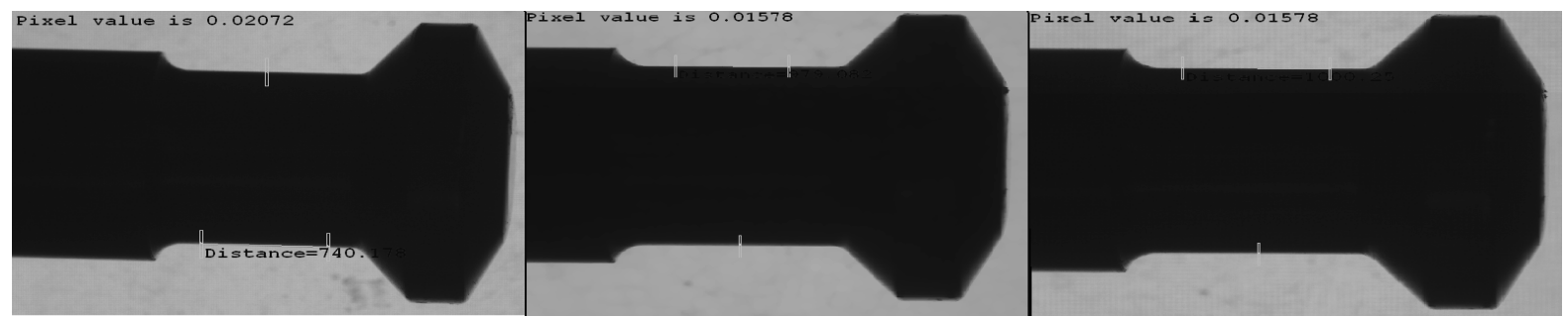

Figure 6. Width of tablet punch image.

Table 1. Result compared.

\begin{tabular}{|c|c|c|c|c|}
\hline & pixel value & $\begin{array}{l}\text { actual value of every } \\
\text { pixel }\end{array}$ & actual value of width & average value of width \\
\hline & 740.178 & $0.02072 \mathrm{~mm}$ & $15.336 \mathrm{~mm}$ & \\
\hline Single image & $\begin{array}{l}765.842 \\
690.223 \\
801.132 \\
787.045\end{array}$ & $\begin{array}{l}0.02003 \mathrm{~mm} \\
0.02222 \mathrm{~mm} \\
0.01899 \mathrm{~mm} \\
0.01949 \mathrm{~mm}\end{array}$ & $\begin{array}{l}15.340 \mathrm{~mm} \\
15.337 \mathrm{~mm} \\
15.210 \mathrm{~mm} \\
15.341 \mathrm{~mm}\end{array}$ & $15.313 \mathrm{~mm}$ \\
\hline & 979.082 & $0.01578 \mathrm{~mm}$ & $15.450 \mathrm{~mm}$ & \\
\hline $\begin{array}{l}\text { Mosaic image of } \\
\text { non-preprocess }\end{array}$ & $\begin{array}{c}901.110 \\
1008.037 \\
880.029 \\
947.501\end{array}$ & $\begin{array}{l}0.01715 \mathrm{~mm} \\
0.01532 \mathrm{~mm} \\
0.01756 \mathrm{~mm} \\
0.01632 \mathrm{~mm}\end{array}$ & $\begin{array}{l}15.453 \mathrm{~mm} \\
15.437 \mathrm{~mm} \\
15.450 \mathrm{~mm} \\
15.461 \mathrm{~mm}\end{array}$ & $15.450 \mathrm{~mm}$ \\
\hline $\begin{array}{l}\text { Mosaic image of mor- } \\
\text { phological processes }\end{array}$ & $\begin{array}{c}1000.250 \\
921.283 \\
1031.527 \\
899.544 \\
969.301\end{array}$ & $\begin{array}{l}0.01578 \mathrm{~mm} \\
0.01715 \mathrm{~mm} \\
0.01532 \mathrm{~mm} \\
0.01756 \mathrm{~mm} \\
0.01632 \mathrm{~mm}\end{array}$ & $\begin{array}{l}15.784 \mathrm{~mm} \\
15.800 \mathrm{~mm} \\
15.803 \mathrm{~mm} \\
15.796 \mathrm{~mm} \\
15.819 \mathrm{~mm}\end{array}$ & $15.800 \mathrm{~mm}$ \\
\hline Standard size & ---- & ---- & $15.880 \mathrm{~mm}$ & $15.880 \mathrm{~mm}$ \\
\hline
\end{tabular}




\section{ACKNOWLEDGEMENTS}

Here, I wish to thank my tutor Professor Haiming Xie, under his careful guidance, I completed the paper and related experiments, thanks my lab colleagues Lu Han, Shijun Guo, and under their help I finished my dissertation fast and accurately.

\section{REFERENCES}

[1] Szeliski, R. (1996) Video mosaics for virtual environments. IEEE Computer Graphics and Applications, 16(2), 22-30.

[2] Peleg, S. and Rousso, B. (2000) Mosaicing on adaptive manifolds. IEEE Transactions on PAMI, 22(10), 1144-1154.

[3] Brown, M. and Lowe, D.G. (2003) Recognising panoramas. Proceedings of IEEE International Conference on Computer Vision, 2(1), 1218-1225.

[4] Mikolajczyk, K. and Schmid, C. (2004) Scale \& Affine invariant interest point detectors. International Journal of Computer Vision, 60(1), 63-86.

[5] Xie, D.H. and Zhan, Z.Q. (2003) Improving Harris corner detector. Journal of Geomatics, 28(2), 22-23.

[6] Harris, C. and Stephens, M.J. (1988) A combined corner and edge detector. Proceedings Fourth Alvey Vision Conference, Manchester, 1988, 147-151.

[7] Qian, W., Fu, Z.Z. and Liu, Q.Q. (2008) Votingstrategy-based approach to image registration. Opto-Electronic Engineering, 35(10), 86-91.

[8] Zhao, W.J., Gong, S.R. and Liu, C.P. (2008) Adaptive Harris corner detection algorithm. Computer Engineering, 34(10), 212-214.

[9] Zhang, J.F. and Xing, X. (2008) Static image registration research based on Harris corner. Science and technology information, 20, 9-10. 